\title{
Continuous matrix product states for coupled fields: Application to Luttinger liquids and quantum simulators
}

\author{
Fernando Quijandría, ${ }^{1}$ Juan José García-Ripoll, ${ }^{2}$ and David Zueco ${ }^{1,3}$ \\ ${ }^{1}$ Instituto de Ciencia de Materiales de Aragón y Departamento de Física de la Materia Condensada, \\ CSIC-Universidad de Zaragoza, Zaragoza, E-50012, Spain \\ ${ }^{2}$ Instituto de Física Fundamental, IFF-CSIC, Serrano 113-bis, Madrid E-28006, Spain \\ ${ }^{3}$ Fundación ARAID, Paseo María Agustín 36, Zaragoza 50004, Spain
}

(Received 16 October 2014; published 23 December 2014)

\begin{abstract}
We present a way of constructing continuous matrix product states (cMPS) for coupled fields. The cMPS is a variational ansatz for the ground state of quantum field theories in one dimension. Our proposed scheme is based on a physical interpretation in which the cMPS class can be produced by means of a dissipative dynamic of a system interacting with a bath. We study the case of coupled bosonic fields. We test the method with previous DMRG results in coupled Lieb-Liniger models. Besides, we discuss a novel application for characterizing the Luttinger liquid theory emerging in the low-energy regime of these theories. Finally, we propose a circuit QED architecture as a quantum simulator for coupled fields.
\end{abstract}

DOI: 10.1103/PhysRevB.90.235142 PACS number(s): 03.65.-w, 05.30.-d, 03.75.Mn, 03.67.Ac

\section{INTRODUCTION}

Quantum information and quantum technologies are providing both a new language and a new experimental landscape for the study of large quantum many-body systems. The study of entanglement in extended lattice models has made it possible to tackle the successful numerical renormalization group (NRG) [1] and the density matrix renormalization group (DMRG) $[2,3]$ and provide them with a solid theoretical background based on the distribution of bipartite entanglement in 1D systems. This understanding made it possible to introduce new methods based on the matrix product states (MPS) formalism that allow studying both static [4] and timedependent phenomena [5-9], together with generalizations for critical [10] and two-dimensional systems [11]. As examples of the success of these methods we can remark the extremely good accuracy of DMRG studies in studying quantum phase transitions of lattice models [3], as well as the success in the quantitative modelization of novel experiments with cold atoms [12-14], ions [15], and photonic systems [16,17].

The above examples rely on lattice models. Sometimes, however, physics is best described via continuum 1D field theories. This includes 1D Bose-Einstein condensates under strong confinement and interaction, long Josephson junctions or nonlinear materials [18-21]. In the seminal work of Verstraete and Cirac [22], the MPS formalism was extended to treat continuum 1D quantum mechanical systems. The continuous matrix product state (cMPS) was formulated as a variational ansatz for obtaining ground states of continuum one-dimensional and nonrelativistic fields [23]. More recently, the cMPS formalism has been used for tackling excited (one-particle) states [24] and $1+1$ relativistic theories $[25,26]$.

In this work, we introduce a natural extension of cMPS states to study coupled fields. We show that thanks to the cMPS formalism, the interaction between fields does not have to be treated perturbatively, developing the appropriate algorithms to compute ground-state properties. This is the main result of the paper and, by itself, it has potential applications to describe systems present experiments with interacting 1D Bose gases [27], as well as the fermion-fermion or fermion-boson interactions, occurring in the so-called ladders $[18,19]$.

A well-known peculiarity for one-dimensional models is their low-energy description as Luttinger liquids [28,29]. These liquids, no matter of the original model, are effective theories of bosonic character and are described by sineGordon-like models. The parameters in the effective theory must be extracted from the original (microscopic) model. In the case of field theories, the parameters are given in terms of the ground state [30,31]. The second important result in this work is that cMPS can be used to derive those Luttinger parameters, both for the single and the coupled field cases.

Finally, we also relate the coupled cMPS ansatz to the simulation of coupled quantum fields. We provide a recipe for building cMPS in the laboratory: engineering discrete quantum systems coupled to transmission lines, as in circuit QED setups. Those laboratory layouts are nothing but prototypes for quantum simulators of field theories within cavity QED [32].

The rest of the paper is organized as follows. The following section, Sec. II, is a (almost) self-contained summary of the cMPS theory. Next, Sec. III is our first application. We use the cMPS, still single field, for obtaining the parameters in the Luttinger liquid theory, explained in Sec. III A and applied to the Lieb-Linniger model, Sec. III B. Section IV explains our extension for coupled fields and Sec. V reports our numerical results for coupled bosonic species. Finally, in Sec. VI, we comment on the application of cMPS for constructing quantum simulators and summarize our results.

\section{OVERVIEW OF CMPS}

We review here the basics of continuous matrix product states (cMPS) for a single field. The cMPS are trial states for a variational estimation of ground states in one-dimensional quantum field theories. We start by considering a quantum system described in the second quantization by means of the field operators $\hat{\psi}(z)$. According to the spin-statistics theorem, these operators must satisfy (anti)commutation relations $\hat{\psi}(z) \hat{\psi}^{\dagger}\left(z^{\prime}\right) \pm \hat{\psi}^{\dagger}\left(z^{\prime}\right) \hat{\psi}(z)=\delta\left(z-z^{\prime}\right)$ according to whether 
they are fermions or bosons. Our system will be defined in a length $L$ with periodic boundary conditions.

The explicit form of the state can be written, as introduced in the seminal work of Verstraete and Cirac [22] $(\hbar=1$ is used through the text),

$$
|\chi\rangle=\operatorname{tr}_{\mathrm{aux}}\left\{\mathcal{P} \mathrm{e}^{\int_{0}^{L} \mathrm{~d} z\left(Q(z) \otimes \mathbb{1}_{\mathcal{F}}+R(z) \otimes \hat{\psi}^{\dagger}(z)\right)}\right\}|\Omega\rangle,
$$

where $\mathcal{P}$ denotes path ordering (we follow a prescription in which, for the argument of the exponential, the value of $z$ increases as we move to the right). $Q(z)$ and $R(z)$ are complex $D \times D$ matrices acting on an auxiliary Hilbert space $\mathcal{H}_{\text {aux }}$. The partial trace $\operatorname{tr}_{\text {aux }}$ is taken over $\mathcal{H}_{\text {aux }}$. The suffix in $\mathbb{1}_{\mathcal{F}}$ emphasizes that it is the identity for the field. Finally, the state $|\Omega\rangle$ is the vacuum of a free theory,

$$
\hat{\psi}(z)|\Omega\rangle=0 .
$$

From now on, we will restrict ourselves to translational invariant setups in which the matrices $R$ and $Q$ become independent of $z$. The cMPS are complete [33], i.e., any one-dimensional quantum field can be casted in the form (1). This class of states can be obtained as the continuum limit of MPS, with bond dimension $D$. The bond dimension can be understood as a measure of the block entanglement. In one dimension, the block entanglement saturates, thus, $D$ is expected to be sufficiently small. If so, we are able to reach any quantum state with a relatively small number of variational parameters $\left(2 D^{2}\right)$. This, combined with the variational method, results in a very powerful technique for finding ground states of one-dimensional theories.

In a relativistic scenario, the block entanglement has a UV logarithmic divergence. This can be understood since the ground state of a relativistic theory, also in $1+1$, will contain zero-point fluctuations from all energy scales, which are the ones contributing more to the entropy [34]. A related argument due to Feynman is quoted in Ref. [25]. The ground state will be dominated by the high-energy contributions. As a consequence, in the variational procedure, the accuracy for describing the low-energy sector is lost. Therefore a cutoff must be introduced. Though challenging, the description of relativistic field theories has been successfully described via cMPS introducing a regularization scheme [25,26]. Here, we will face the most favorable case of one-dimensional nonrelativistic theories.

Inherited from their discrete countenparts, the cMPS is not unique but the gauge transformation $Q \rightarrow g Q g^{-1}$ and $R \rightarrow$ $g R g^{-1}$ leaves the state $|\chi\rangle$ invariant [23]. It turns out that the gauge

$$
Q+Q^{\dagger}+R^{\dagger} R=0
$$

is quite convenient. In this gauge, the cMPS state (1) can be rewritten as

$$
|\chi\rangle=\operatorname{tr}_{\mathrm{aux}}\{U(L, 0)\}|\Omega\rangle
$$

with

$$
U(L, 0)=\mathcal{P} \mathrm{e}^{-i \int_{0}^{L} \mathrm{~d} z\left(K \otimes \mathbb{1}_{\mathcal{F}}+i R \otimes \hat{\psi}^{\dagger}(z)-i R^{\dagger} \otimes \hat{\psi}(z)\right)}
$$

and, $K=K^{\dagger}$ Hermitian,

$$
Q=-i K-\frac{1}{2} R^{\dagger} R
$$

(a)

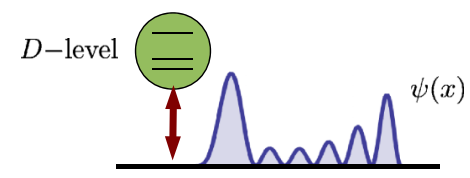

(b)

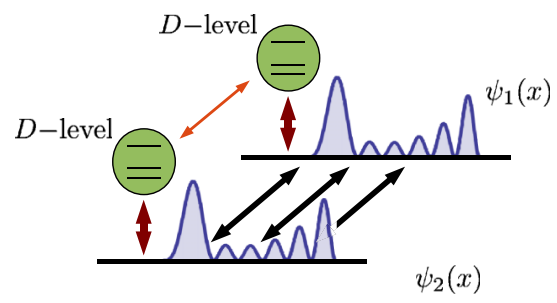

FIG. 1. (Color online) (a) The cMPS can be built by letting an ancilla (green circle in the figure) be coupled to a continuous field $[\psi(x)]$. (b) In a similar way, we propose that coupled fields can also be constructed by coupling independent ancillas.

which implies the gauge condition (3). The unitary operator $U$, in Eq. (5), is formally equivalent to a evolution in $z$ time for the field $\hat{\psi}(z)$ and a $D$-level (auxiliary) system with Hamiltonian $K$. Field and ancilla are coupled via $i R \otimes \hat{\psi}^{\dagger}(z)-i R^{\dagger} \otimes$ $\hat{\psi}(z)$. The ground state is described in terms of the matrices $K$ and $R$, i.e., in terms of an auxiliary zero-dimensional system. This suggests an holographic interpretation for the cMPS [35]. See Fig. 1(a) for a pictorial interpretation.

It remains to provide operational rules for computing within the cMPS formalism. To be precise, we must be able to write any field observable $\left\langle\chi\left|O\left(\hat{\psi}, \hat{\psi}^{\dagger}\right)\right| \chi\right\rangle$ in terms of the matrices $R$ and $Q$. As detailed in Ref. [36], the following relations are found:

$$
\begin{aligned}
\langle\chi \mid \chi\rangle & =\operatorname{tr}\left\{\mathrm{e}^{T L}\right\}, \\
\left\langle\hat{\psi}^{\dagger}(z) \hat{\psi}(z)\right\rangle= & \operatorname{tr}\left\{\mathrm{e}^{T L}\left(R \otimes R^{*}\right)\right\}, \\
\left\langle\partial_{z} \hat{\psi}^{\dagger}(z) \partial_{z} \hat{\psi}(z)\right\rangle= & \operatorname{tr}\left\{\mathrm{e}^{T L}\left([Q, R] \otimes\left[Q^{*}, R^{*}\right]\right)\right\},
\end{aligned}
$$

$$
\left\langle\hat{\psi}^{\dagger}(z) \hat{\psi}^{\dagger}(z) \hat{\psi}(z) \hat{\psi}(z)\right\rangle=\operatorname{tr}\left\{\mathrm{e}^{T L}\left(R R \otimes R^{*} R^{*}\right)\right\},
$$

here,

$$
T=Q \otimes \mathbb{1}+\mathbb{1} \otimes Q^{*}+R \otimes R^{*} .
$$

The Kronecker products in the ancilla space occur because some identities, e.g., $\operatorname{tr}\{A\} \operatorname{tr}\{B\}=\operatorname{tr}\{A \otimes B\}$, have been used.

To avoid those products in the auxiliary space, the isomorphism $|a\rangle|b\rangle \rightarrow|a\rangle\left\langle b^{*}\right|$ is introduced. This allows us to map vectors in $\mathcal{H}_{\text {aux }} \otimes \mathcal{H}_{\text {aux }}$ into operators acting on $\mathcal{H}_{\text {aux }}$. This can be understood from the fundamental property

$$
\begin{aligned}
& \operatorname{tr}\left\{\sum_{i} \sum_{a b c d} A_{i} \otimes B_{i}^{*}|a\rangle|b\rangle\langle c|\langle d|\right\} \\
& =\operatorname{tr}\left\{\sum_{i} \sum_{a b c d}\left|d^{*}\right\rangle\left\langle c\left|A_{i}\right| a\right\rangle\left\langle b^{*}\right| B_{i}^{\dagger}\right\} .
\end{aligned}
$$

The former also implies that operators acting on $\mathcal{H}_{\text {aux }}$ are mapped into superoperators. Therefore the action of $T$ on a ket $|\rho\rangle$ will be mapped into $\mathcal{T}[\rho]$, where $\mathcal{T}$ is a superoperator acting on the state (matrix) $\rho$. Under the isomorphism, it is 
straightforward to show that

$$
\mathcal{T}[\varrho]:=-i[K, \varrho(z)]+R \varrho R^{\dagger}-\frac{1}{2}\left[R^{\dagger} R, \varrho(z)\right]_{+} \cdot
$$

This is nothing but the dissipator governing a Linblad-like evolution $\mathrm{d}_{z} \varrho=\mathcal{T} \varrho$ for the irreversible dynamics of a system coupled to a reservoir. In this case, the role of the system is being played by the ancilla and that of the bath by the field [see Fig. 1(a) and the discussion above on the holographic interpretation]. The Linbladian is a positive-semidefinite operator, $\mathcal{T} \leqslant 0$, having at least one zero eigenvalue [37]. With this at hand, Eq. (7) can be rewritten as

$$
\langle\chi \mid \chi\rangle=\left\langle l \mid \mathrm{e}^{T L} r\right\rangle=\operatorname{Tr}\left(\mathrm{e}^{T L}|r\rangle\langle l|\right)=\operatorname{Tr}\left(l \mathrm{e}^{\mathcal{T} L} r\right) .
$$

Here, $\langle l|$ and $|r\rangle$ are the left and right eigenvectors of $T$ (respectively) associated with its zero eigenvalue. We have assumed implicitly the limit $L \rightarrow \infty$ where this eigenvalue yields the principal contribution to the exponential. In the third equality, the above introduced isomorphism has been used. Note that the zero eigenvectors of $T$, under the isomorphism, are mapped into the stationary solutions of the Linblad equation (left and right equations). Accordingly, the action of $T$ into the bra $\langle l|$ can also be mapped into the action of a superoperator on a matrix: $\langle l| T \Leftrightarrow Q^{\dagger} l+\varrho_{l} Q+R^{\dagger} l R$. It is easy to see that, under the gauge (3), $l^{*}=\mathbb{1}$ is a solution of the stationary Linblad-like dynamics $\left(d_{z} l^{*}=0\right)$. Combining all of this, we end up with

$$
\langle\chi \mid \chi\rangle=\operatorname{tr}\left(r^{*}\right)=1,
$$

where $d_{z} r^{*}=0$.

In a similar way, we can re-express the expectation value of any operator in terms of the steady-state solution $\varrho^{*}$ of the right Linblad equation:

$$
\begin{aligned}
\left\langle\hat{\psi}^{\dagger}(z) \hat{\psi}(z)\right\rangle & =\operatorname{tr}\left(R^{\dagger} R \varrho^{*}\right), \\
\left\langle\partial_{z} \hat{\psi}^{\dagger}(z) \partial_{z} \hat{\psi}(z)\right\rangle & =\operatorname{tr}\left([Q, R]^{\dagger}[Q, R] \varrho^{*}\right), \\
\left\langle\hat{\psi}^{\dagger}(z) \hat{\psi}^{\dagger}(z) \hat{\psi}(z) \hat{\psi}(z)\right\rangle & =\operatorname{tr}\left(\left(R^{\dagger}\right)^{2} R^{2} \varrho^{*}\right) .
\end{aligned}
$$

With this, we conclude our overview of the cMPS formalism. In the limit $L \rightarrow \infty$, we will be concerned with the groundstate energy density $e_{0}=\left\langle\chi\left|\hat{\mathcal{H}}\left(\psi, \psi^{\dagger}\right)\right| \chi\right\rangle$ (where $\hat{\mathcal{H}}$ is the Hamiltonian density operator). The latter can be computed by minimizing with the matrices $R$ and $Q$ as input and using the latter relations (and similar ones). Once the minimization procedure has finished, observables can be computed with the same relations using the optimized matrices.

\section{APPLICATION TO LUTTINGER LIQUIDS}

\section{A. Bosonization}

At low temperatures, a large class of one-dimensional theories exhibit excitations of bosonic nature and their correlation functions are characterized by power laws. An interesting feature of $1 \mathrm{D}$ is that this class makes almost no distinction between bosons and fermions. Haldane [28,29] termed this class of theories Luttinger liquids. The bosonic nature of the low-energy excitations in 1D is due to the enhanced role quantum fluctuations acquire in low-dimensional systems.

For a given microscopic model, the so-called bosonization prescription, consists in expressing the original degrees of freedom in terms of new fields, which capture the collective behavior characterizing the low-energy regime. For the case of a bosonic field, we will introduce the density-phase representation [18]

$$
\hat{\psi}^{\dagger}(x)=\sqrt{\hat{\rho}(x)} \mathrm{e}^{-i \hat{\theta}(x)},
$$

where $\hat{\rho}(x):=\psi^{\dagger}(x) \psi(x)$ is the particle density field and $\hat{\theta}(x)$ the phase field. Close enough to the ground state, we can safely approximate the density operator by

$$
\hat{\rho}(x) \sim \rho_{0}-\frac{1}{\pi} \partial_{x} \hat{\phi}(x),
$$

where $\rho_{0}$ is the ground-state density and the operator $\hat{\phi}(x)$ characterizes the fluctuations over the ground state. The commutation relations for bosonic fields will translate into a canonical commutation relation for the $\hat{\theta}$ and $\hat{\phi}$ fields:

$$
\left[\frac{1}{\pi} \partial_{x} \hat{\phi}(x), \hat{\theta}\left(x^{\prime}\right)\right]=-i \delta\left(x-x^{\prime}\right) .
$$

\section{B. Calculation for the Lieb-Liniger model}

We are going to apply the previous ideas to the Lieb-Liniger model [38]. The former describes a 1D nonrelativistic bosonic gas interacting via a repulsive zero-range potential:

$$
\hat{H}=\int_{0}^{L} \mathrm{~d} x \frac{1}{2 M} \partial_{x} \hat{\psi}^{\dagger}(x) \partial_{x} \hat{\psi}(x)+c\left[\hat{\psi}^{\dagger}(x)\right]^{2}[\hat{\psi}(x)]^{2} .
$$

The Lieb-Liniger model is exactly solvable by means of a Bethe ansatz. In fact, the solution shows that at low energies, this model displays a Luttinger liquid behavior [39]. An excellent agreement between the exact ground-state energy density and the cMPS solution has already been provided [22]. Finally, note that this model conserves the particle number density. This quantity will represent a minimization constraint when finding the ground state numerically.

Following the bosonization scheme, the effective Hamiltonian describing the low-energy behavior of the Lieb-Liniger model is

$$
\hat{H}_{\text {eff }}=\frac{v}{2 \pi} \int_{0}^{L} \mathrm{~d} x K\left[\partial_{x} \hat{\phi}(x)\right]^{2}+\frac{1}{K}\left[\partial_{x} \hat{\theta}(x)\right]^{2} .
$$

Hence, the low-energy regime can be completely characterized by means of two parameters (Luttinger parameters): the velocity $v$ and the dimensionless parameter $K$. These, in turn, can be related to the ground state energy density $e_{0}(\rho)$ of the microscopic Hamiltonian (21). The corresponding relations are [30]

$$
\begin{gathered}
v^{2}=\left.\frac{\rho_{0}}{M} \frac{\partial^{2} e_{0}}{\partial \rho^{2}}\right|_{\rho=\rho_{0}}, \\
K^{2}=\frac{\pi^{2} \rho_{0}}{M}\left(\left.\frac{\partial^{2} e_{0}}{\partial \rho^{2}}\right|_{\rho=\rho_{0}}\right)^{-1} .
\end{gathered}
$$

It is possible to obtain asymptotic (analytic) expressions for the former parameters in terms of the dimensionless coupling constant $\gamma=M c / \rho_{0}$. In Fig. 2, we compare those asymptotic limits (small and large repulsion, see Ref. [30]) for $v$ and $K$ with (23) and (24) as obtained from the ground-state energy density computed with cMPS. We have performed simulations 


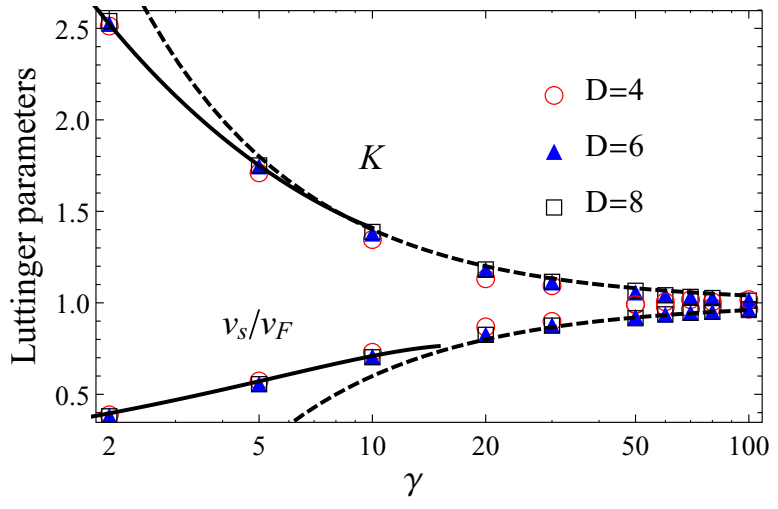

FIG. 2. (Color online) Luttinger parameters as a function of the dimensionless zero-range interaction constant $\gamma=M c / \rho_{0}$ for the single LL model, Eq. (21) (we choose $\rho_{0}=1$ and $M=1 / 2$ ). The full and dashed lines correspond to the weak and strong repulsion limits, respectively. These are compared with our cMPS results for bond dimensions $D=4$ (open circles), 6 (filled triangles), and 8 (open squares).

for $D=2,4,6$, and 8 . For every bond dimension, the groundstate energy density was calculated for up to twelve different densities. By interpolation, we constructed the continuous function $e_{0}(\rho)$ and the derivatives were calculated from it. Results show that for a moderately small bond dimension $(D=$ 6 ), it is possible to match the predicted asymptotic behavior up to high values of $\gamma$. Results for $D=2$ are not shown for the sake of clarity (such a small bond dimension does not capture correctly the ground state of the Lieb-Liniger model).

\section{EXTENSION/GENERALIZATION FOR COUPLED FIELDS}

The cMPS formalism can be naturally extended to treat a multispecies system. Let us consider a system of length $L$ in which coexist $q$ bosonic and/or fermionic particle species, which are annihilated by the operators $\hat{\psi}_{\alpha}, \alpha=1, \ldots, q$. These operators satisfy (anti)commutation relations

$$
\begin{aligned}
& \hat{\psi}_{\alpha}(x) \hat{\psi}_{\beta}\left(x^{\prime}\right)-\eta_{\alpha \beta} \hat{\psi}_{\beta}\left(x^{\prime}\right) \hat{\psi}_{\alpha}(x)=0, \\
& \hat{\psi}_{\alpha}(x) \hat{\psi}_{\beta}^{\dagger}\left(x^{\prime}\right)-\eta_{\alpha \beta} \hat{\psi}_{\beta}^{\dagger}\left(x^{\prime}\right) \hat{\psi}_{\alpha}(x)=\delta_{\alpha \beta} \delta\left(x-x^{\prime}\right),
\end{aligned}
$$

where $\eta_{\alpha \beta}=+1$ if at least one of the fields $\alpha$ or $\beta$ is bosonic and $\eta_{\alpha \beta}=-1$ if both fields are of fermionic nature.

The $q$ species cMPS state is defined as [23]

$$
|\chi\rangle=\operatorname{tr}_{\mathrm{aux}}\left\{\mathcal{P} \mathrm{e}^{\int_{0}^{L} \mathrm{~d} z \tilde{Q}(z) \otimes \mathbb{1}+\sum_{\alpha=1}^{q} \tilde{R}_{\alpha}(z) \otimes \hat{\psi}_{\alpha}^{\dagger}(z)}\right\}|\Omega\rangle,
$$

here, matrices $\tilde{R}_{\alpha}$ have been introduced for each one of the fields $\hat{\psi}_{\alpha}$ and a single Hamiltonian $\tilde{K}$ for the auxiliary system. We will employ the tilde notation for the variational parameters of the multispecies cMPS state to differentiate them from their single field counterparts, cf. Eq. (1). The matrix $\tilde{Q}$ is now defined as

$$
\tilde{Q}(x)=-i \tilde{K}(x)-\frac{1}{2} \sum_{\alpha=1}^{q} \tilde{R}_{\alpha}^{\dagger}(x) \tilde{R}_{\alpha}(x) .
$$

At difference with the single field case, a regularity condition must be imposed on the $\tilde{R}_{\alpha}$ matrices in order that the expectation value of the nonrelativistic kinetic energy, as computed with (27), will not become divergent. This condition reads

$$
\tilde{R}_{\alpha}(x) \tilde{R}_{\beta}(x)-\eta_{\alpha \beta} \tilde{R}_{\beta}(x) \tilde{R}_{\alpha}(x)=0 .
$$

In other words, the matrices $\tilde{R}_{\alpha}$ inherit the (anti)commutation relation of their corresponding fields. With these ideas in mind we can extend the operational rules for computing expectation values with cMPS. For example,

$$
\langle\chi \mid \chi\rangle=\operatorname{tr}\left\{\mathrm{e}^{\tilde{T} L}\right\},
$$

where the transfer operator (11) has been generalized to

$$
\tilde{T}=\tilde{Q} \otimes \mathbb{1}+\mathbb{1} \otimes \tilde{Q}^{*}+\sum_{\alpha=1}^{q} \tilde{R}_{\alpha} \otimes \tilde{R}_{\alpha}^{*}
$$

and translational invariance has been assumed for simplicity.

Special care must be taken into account for systems where two or more fermionic species coexist. Let us discuss correlators like $\left\langle\hat{\psi}_{\alpha}^{\dagger}(x) \hat{\psi}_{\beta}(y)\right\rangle$. Expanding the path-ordered exponential in (27), which acts on the vacuum $|\Omega\rangle$ of the field theory, and taking the annihilation operators to the right (normal ordering prescription), we obtain [23]

$$
\left\langle\hat{\psi}_{\alpha}^{\dagger}(x) \hat{\psi}_{\beta}(y)\right\rangle=\operatorname{tr}\left\{\mathrm{e}^{\tilde{T} y}\left(\tilde{R}_{\beta} \otimes \mathbb{1}\right) \mathrm{e}^{\tilde{T}_{\alpha}(x-y)}\left(\mathbb{1} \otimes \tilde{R}_{\alpha}^{*}\right) \mathrm{e}^{\tilde{T}(L-x)}\right\},
$$

$(x>y)$ where the generalized transfer operator $\tilde{T}_{\alpha}$ deals with the exchange statistics

$$
\tilde{T}_{\alpha}=\tilde{Q} \otimes \mathbb{1}+\mathbb{1} \otimes \tilde{Q}^{*}+\sum_{\beta=1}^{q} \eta_{\alpha \beta} \tilde{R}_{\beta} \otimes \tilde{R}_{\beta}^{*}
$$

For the case of bosonic systems, $\tilde{T}_{\alpha}=\tilde{T}$. The transfer operator $\tilde{T}$ governs the evolution of states in the ancillary space. Similarly to the single field case, this evolution can be mapped to a dissipative dynamics corresponding to the following Linblad quantum master equation:

$$
\frac{d \tilde{\varrho}(z)}{d z}=-i[\tilde{K}, \tilde{\varrho}(z)]+\sum_{\alpha=1}^{q} \tilde{R}_{\alpha} \tilde{\varrho} \tilde{R}_{\alpha}^{\dagger}-\frac{1}{2}\left[\tilde{R}_{\alpha}^{\dagger} \tilde{R}_{\alpha}, \tilde{\varrho}(z)\right]_{+} \cdot
$$

Thus we have again the picture of the ancilla coupled to a bath (the fields) by means of the operators $\tilde{R}_{\alpha}$.

Consider now the case of two bosonic fields $\hat{\psi}_{1}$ and $\hat{\psi}_{2}$. We are interested in studying how the matrices $\left(\tilde{R}_{\alpha}\right.$ and $\left.\tilde{K}\right)$, which define the cMPS state in this two-species system, can be constructed from the matrices which characterize a single field. The simplest scenario considers two uncoupled fields. We have seen how the problem of computing expectation values in the ground-state can be reduced to a dissipative dynamics going on in the auxiliary space-where the state of the total auxiliary system is described in terms of the density matrix $\tilde{\rho}$. In the absence of a coupling between the fields, we should be able to recover our single field solutions. This is nothing but to demand the density matrix to be separable, that is, $\widetilde{\varrho}=\varrho_{1} \otimes \varrho_{2}$. Both fields do not need to be identical, therefore, each of them will have associated a different set of matrices $R_{\alpha}$ and $K_{\alpha}$ which act on the corresponding auxiliary space $\mathcal{A}_{\alpha}$. For simplicity, 
we assume that both $\mathcal{A}_{1}$ and $\mathcal{A}_{2}$ have the same bond dimension $D_{1}=D_{2}=D$. The total auxiliary space for the two fields will be the tensor product of the individual spaces $\tilde{\mathcal{A}}=\mathcal{A}_{1} \otimes \mathcal{A}_{2}$. Due to the tensor product structure, the bond dimension of the total auxiliary space is now $\tilde{D}=D^{2}$. The ancillas evolve independently according to the total Hamiltonian

$$
\tilde{K}=K_{1} \otimes \mathbb{1}+\mathbb{1} \otimes K_{2} .
$$

Similarly, each auxiliary system will couple to its quantum field by means of the matrices $R_{\alpha}$. The extension of these to the product space is

$$
\begin{gathered}
\tilde{R}_{1}=R_{1} \otimes \mathbb{1}, \\
\tilde{R}_{2}=\mathbb{1} \otimes R_{2} .
\end{gathered}
$$

Notice that the matrices $\tilde{R}_{\alpha}$ satisfy the bosonic commutation relation $\left[\tilde{R}_{1}, \tilde{R}_{2}\right]=0$ as it is demanded for a multispecies system (29). As desired, our construction lets us recover the results for single fields. For instance, $\left\langle\hat{\psi}_{1}^{\dagger} \hat{\psi}_{1}\right\rangle=\operatorname{tr}\left(\tilde{\rho}^{*} \tilde{R}_{1}^{\dagger} \tilde{R}_{1}\right)=\operatorname{tr}\left(\rho_{1}^{*} R_{1}^{\dagger} R_{1}\right) \operatorname{tr} \rho_{2}^{*}=\operatorname{tr}\left(\rho_{1}^{*} R_{1}^{\dagger} R_{1}\right)$ (as $\operatorname{tr} \rho_{2}^{*}=1$ for a density matrix).

How is this picture modified in the presence of a coupling between $\hat{\psi}_{1}$ and $\hat{\psi}_{2}$ ? An arbitrary operator $\tilde{C}$, mapping $\tilde{\mathcal{A}}$ into itself, can be represented as $\tilde{C}=\sum_{i} c_{i} A_{i} \otimes B_{i}$, where $A_{i}$ acts on $\mathcal{A}_{1}$ and $B_{i}$ acts on $\mathcal{A}_{2}$. Therefore this defines the most general structure for the matrices $\tilde{R}_{\alpha}$ and $\tilde{K}$. Those general matrices must satisfy the regularity conditions (29), which complicates their construction. A possible solution is the following. We use the intuitive interpretation for the cMPS in terms of a system bath, see Fig. 1(b) and the discussion on the holographic interpretation below Eq. (13). Starting from the decoupled solution (35), (36), and (37), we switch on the coupling adiabatically and expect that our solutions will start to modify. This is depicted schematically in Fig. 1(b). Here, as one introduces the coupling between the physical fields, the individual auxiliary spaces will also start to interact . Inspired by this procedure, we propose the following construction in the presence of a coupling. First of all, the matrices $\tilde{R}_{1}$ and $\tilde{R}_{2}$ will continue to be described by (36) and (37), respectively. In this way, we guarantee that they commute, satisfying (29) trivially. In order to render the state nonseparable, the matrix $\tilde{K}$ is written in a general way but containing the uncoupled solution as a limit (35). This is done as follows:

$$
\tilde{K}=K_{1} \otimes \mathbb{1}+\mathbb{1} \otimes K_{2}+\sum_{p=0}^{P} Z_{1}^{(p)} \otimes Z_{2}^{(p)},
$$

where $Z_{1}^{(0)}=Z_{2}^{(0)}=0$. In order to keep $\tilde{K}$ Hermitian, we will demand that the matrices $Z_{i}^{(p)}$ are Hermitian too. The number $P$ of pairs of $Z$ matrices is arbitrary. In principle, we will expect it to grow with the strength of the coupling.

We have seen for the single field case, that the cMPS ansatz is able to map the properties of a continuous one-dimensional field theory by means of $2 D^{2}$ variational parameters (with of course, a relatively small bond dimension $D$ ). Doubling the number of fields, as well as introducing $P$ pairs of the already defined $Z$ matrices, increases the total number of variational parameters to $(4+2 P) D^{2}$.

\section{TWO-SPECIES BOSONIC SYSTEM}

The system we have in mind to test the cMPS method for coupled fields is a two-component bosonic system. Binary systems of this kind (as well as bosonic + fermionic mixtures) are Luttinger liquids with a rich phase diagram [40,41]. We will consider two Lieb-Liniger gases with a density density coupling. This is described by the following Hamiltonian:

$$
\begin{aligned}
\hat{H}= & \frac{1}{2 M} \sum_{\alpha=1}^{2} \int_{0}^{L} \mathrm{~d} x \partial_{x} \hat{\psi}_{\alpha}^{\dagger}(x) \partial_{x} \hat{\psi}_{\alpha}(x) \\
& +c \sum_{\alpha=1}^{2} \int_{0}^{L} \mathrm{~d} x\left(\hat{\psi}_{\alpha}^{\dagger}(x)\right)^{2}\left(\hat{\psi}_{\alpha}(x)\right)^{2}+g \int_{0}^{L} \mathrm{~d} x \hat{\rho}_{1}(x) \hat{\rho}_{2}(x) .
\end{aligned}
$$

In order to obtain the low-energy behavior of this model, we will use the bosonization technique introduced in Sec. III A. As already explained, this consists in rewriting the bosonic fields in terms of the collective fields $\hat{\theta}_{\alpha}$ and $\hat{\phi}_{\alpha}$ which characterize the bosonic low-energy excitations. Hamiltonian (39) conserves the individual particle densities, $\left[\hat{H}, \hat{\rho}_{\alpha}\right]=[\hat{H}, \hat{\rho}]=$ $0(\alpha=1,2)$. Therefore we can fix these two densities as minimization constraints in the cMPS procedure. In Eq. (19), we have considered the lowest order term in a harmonic expansion of the density operator. A more careful treatment[18] shows that, the correct expansion for the density operator in terms of the field $\hat{\phi}_{\alpha}$ is of the form $\hat{\rho}_{\alpha}(x)=\left[\rho_{0 \alpha}-\right.$ $\left.\partial_{x} \hat{\phi}_{\alpha}(x) / \pi\right] \sum_{p} \mathrm{e}^{i 2 p\left(\pi \rho_{0 \alpha} x-\hat{\phi}_{\alpha}(x)\right)}$. Our former simplification is justified due to the fact that, at long distances (low energies), the phase terms oscillate very fast and will average to zero upon integration. In performing the bosonization, we must retain the most dominant terms at low energies. For the case of our interspecies coupling, this supposes to consider also the first harmonic $p=1$. This leads, at low temperatures, to a coupling contribution of the form: $1 / 2 \pi \int \mathrm{d} x\left[2 g_{x} \partial_{x} \hat{\phi}_{1} \partial \hat{\phi}_{2}+\right.$ $\left.g_{c} \cos \left(2\left(\hat{\phi}_{1}-\hat{\phi}_{2}\right)+\pi \delta x\right)\right]\left(\right.$ with $\left.\delta=\rho_{01}-\rho_{02}\right)$. Of particular interest for us will be the case of equal filling $\rho_{01}=\rho_{02}$ $(\delta=0)$. Species 1 and 2 in the low-energy effective Hamiltonian can be decoupled by introducing the normal modes $\hat{\phi}_{+}=1 / \sqrt{2}\left(\hat{\phi}_{1}+\hat{\phi}_{2}\right)$ and $\hat{\phi}_{-}=1 / \sqrt{2}\left(\hat{\phi}_{1}-\hat{\phi}_{2}\right)$. In terms of these, we have that the low-energy excitations of (39) can be described by the effective Hamiltonian

$$
\begin{aligned}
\hat{H}_{\text {eff }}= & \frac{1}{2 \pi} \int \mathrm{d} x\left[\sum_{\nu= \pm} v_{\nu}\left(\left(\partial_{x} \hat{\phi}_{\nu}\right)^{2}+\frac{1}{K_{v}}\left(\partial_{x} \hat{\theta}_{\nu}\right)^{2}\right)\right. \\
& \left.+g_{c} \cos \left(\sqrt{8} \hat{\phi}_{-}\right)\right] .
\end{aligned}
$$

Similarly to the single field case, the Luttinger parameters $v_{ \pm}$and $K_{ \pm}$can be related to the ground-state energy density $e_{0}\left(\rho_{+}, \rho_{-}\right)$(as a function of the normal densities) of Hamiltonian (39):

$$
\begin{gathered}
v_{ \pm}^{2}=\left.\frac{2 \rho_{0 \pm}}{M} \frac{\partial^{2} e_{0}}{\partial \rho_{ \pm}^{2}}\right|_{\rho_{ \pm}=\rho_{0 \pm}} . \\
K_{ \pm}^{2}=\frac{\pi^{2} \rho_{0 \pm}}{2 M}\left(\left.\frac{\partial^{2} e_{0}}{\partial \rho_{ \pm}^{2}}\right|_{\rho_{ \pm}=\rho_{0 \pm}}\right)^{-1} .
\end{gathered}
$$



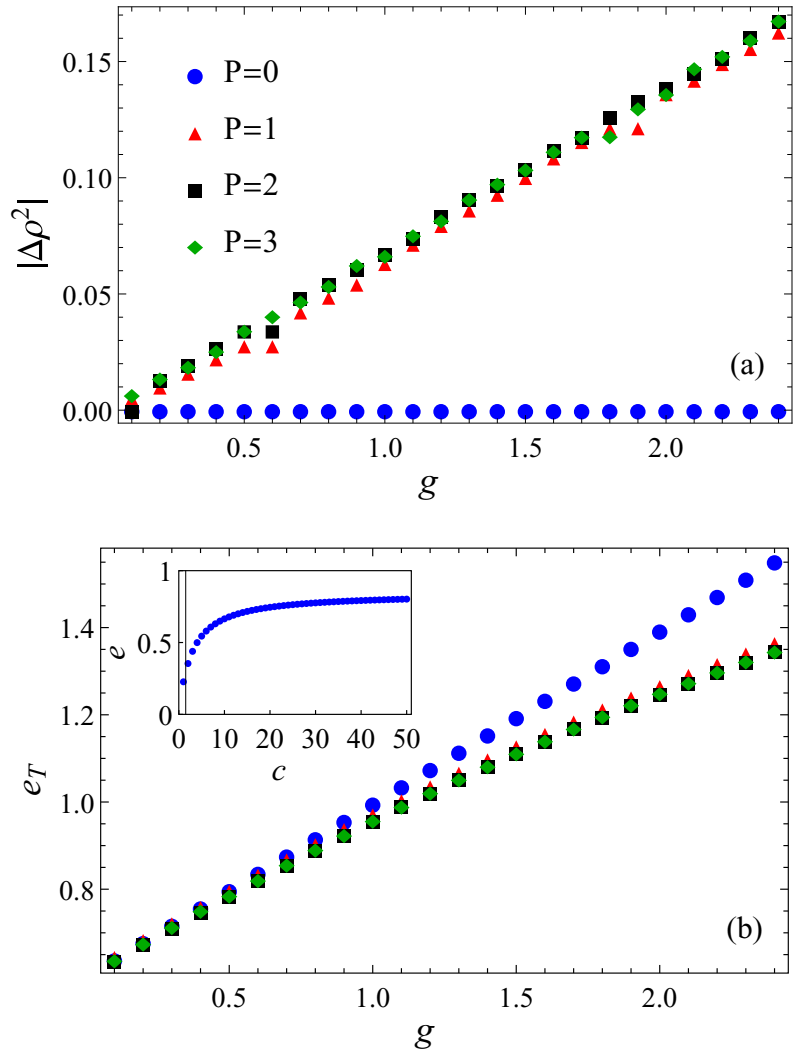

FIG. 3. (Color online) (a) Ground-state density-density correlations and (b) total energy density for the coupled LL model, Hamiltonian (39). Both as a function of the interspecies coupling strength $g$ for equal densities $\rho_{01}=\rho_{02}=0.63$ and $c=1.5$. The ancilla space for each field has bond dimension $D$ equal to 6 . We couple the ancillas by means of $P$ pairs of $Z$ matrices. Results are shown for $P=0$ (circles), 1 (triangles), 2 (squares) and 3 (diamonds). (Inset) Ground-state energy density for a single Lieb-Liniger chain as a function of $c(D=6)$. The vertical line denotes the value of $c$ at which we are coupling two fields.

Coupled species have been thoroughly studied [40,41]. In this work we study coupled bosonic species described by (39). The range of parameters considered coincides with the one in Refs. $[42,43]$ where a DMRG study is reported, hence a direct comparison is possible.

In Fig. 3(a), we plot the ground-state density-density correlations $\left|\Delta \rho^{2}\right|=\left|\left\langle\hat{\rho}_{1}(x) \hat{\rho}_{2}(x)\right\rangle-\left\langle\hat{\rho}_{1}(x)\right\rangle\left\langle\hat{\rho}_{2}(x)\right\rangle\right|$ as a function of the interspecies coupling $g$ for different values of $P$. Both, the repulsion strength $c$ and the bond dimension $D$ are kept fixed ( $c=1.5$ and $D=6)$. As expected, $P=0$ renders the state separable and no correlations are observed. Making $P \neq 0$, the correlations between the two fields build up. They grow with the coupling strength. In this range of parameters, $P=2$ seems to be sufficient for account with the physics.

The ground-state energy density as a function of $g$ is shown in Fig. 3(b). Only the last term of (39) depends on $g$, therefore, (for a fixed value of $c$ ) the first two terms yield a constant contribution. The case $P=0$ yields a mean-field treatment where the interaction is replaced by $g\langle\hat{\rho} 1\rangle\left\langle\hat{\rho}_{2}\right\rangle$. It was already mentioned that $\left[\hat{H}, \hat{\rho}_{1}\right]=\left[\hat{H}, \hat{\rho}_{2}\right]=0$. Thus, with $P=0$, the energy as a function of $g$ has a linear dependence with slope
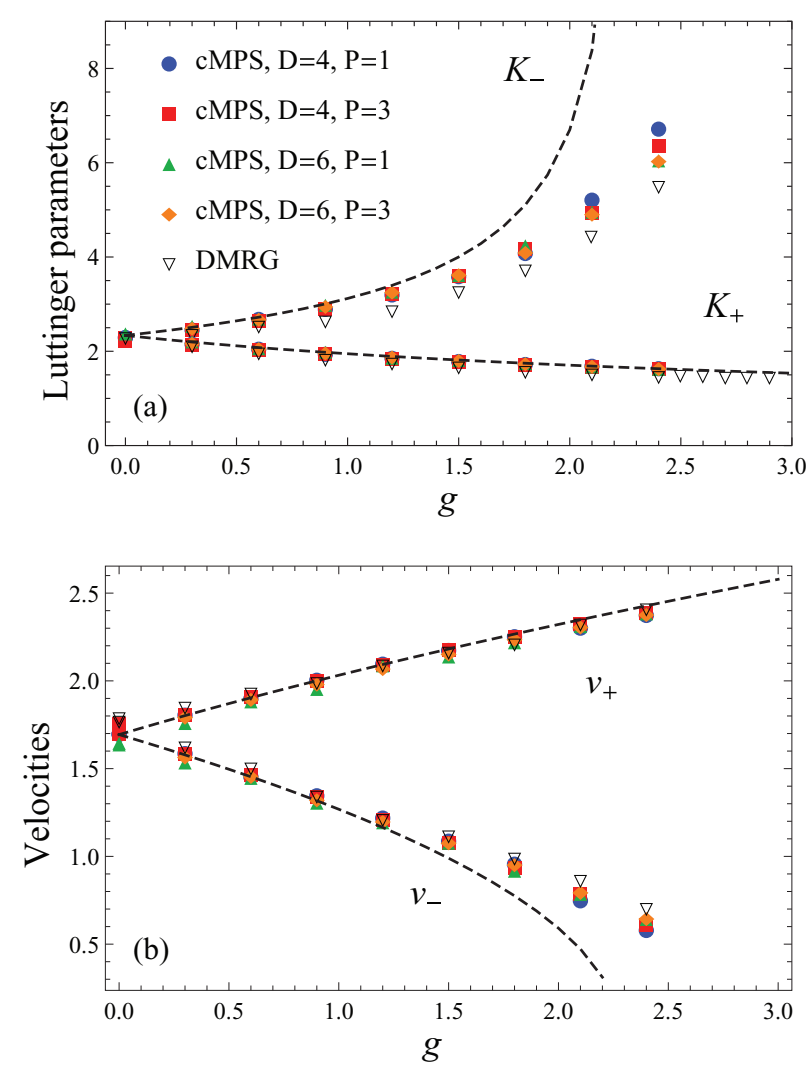

FIG. 4. (Color online) Charge (+) and spin(-) Luttinger parameters as a function of the coupling $g$ between fields. Here we show a comparison between a weak-coupling approximation (dashed line), DMRG [42,43], and our results implementing cMPS for coupled fields with different bond dimensions and values of the parameter $P$ for fixed $c=1.5$. (a) $K_{ \pm}$(b) velocities $v_{ \pm}$.

$\rho_{01} \rho_{02}$. Including quantum correlations $(P \neq 0)$, the energy is not longer a linear function of the coupling, as seen in Fig. 3(b).

As it was already discussed, the low-energy description of our model is characterized by the Luttinger parameters (41) and (42). In particular, the difference in normal velocities $v_{ \pm}$yields the charge-spin separation-a typical experimental characteristic in mixtures. In Fig. 4, we compare for both the $v_{ \pm}$and $K_{ \pm}$our cMPS results with the DMRG values extracted from Refs. [42,43]. Let us remark the excellent agreement in $K_{+}$and $v_{+}$and the minor discrepancy in $K_{-}$or $v_{-}$. The very small differences could be attributed to a number of issues: small bond dimension in the cMPS $(D=6)$ to be compared with the DMRG (several hundreds), or the fact that the DMRG theory is discretized an the cMPS is fully continuous.

\section{QUANTUM SIMULATION OF COUPLED CMPS}

There exist two approaches towards the quantum simulation of continuous or discrete field theories [44]. The conventional one consists on taking a flexible quantum system, such as a Bose-Einstein condensate, ultracold atoms in an optical lattice or a superconductor, and working with it to implement the full field theory, or an approximate version of it, in the experiment. This "analogue" quantum simulator therefore evolves and 
equilibrates as the original model dictates and all observables may be directly studied on the experiment itself.

A second possibility for quantum simulation arises from the physical interpretation of cMPS. The idea is that there exists a mapping between a continuous matrix product state and a physical process operating on a small quantum-mechanical object. This mapping between states and channels was already evidenced for discrete MPS [45,46] and has been recently generalized for cMPS [32], by means of their physical interpretation in terms of a system (the ancilla) coupled to a bath (the field). The beauty of this mapping is that it is quite general and applies to a variety of quantum optical systems. The prototypical system is an atom-cavity setup (the system or ancilla in the language of this paper) that interacts with external input and output fields through the bath (the field in cMPS) in this case the electromagnetic field. However, any other quantum discrete system coupled to an outer field, where different order correlations of the latter can be measured, such as circuit QED [47,48] would do the job.

Let us now summarize the proposal in Ref. [32]. The atomcavity system is described through the well known JaynesCummings (JC) model,

$$
\hat{H}_{\text {ancilla }}=\hat{H}_{\mathrm{JC}}=g\left(\hat{a}^{\dagger} \hat{\sigma}^{-}+\text {H.c. }\right)+\Omega\left(\sigma^{+}+\sigma^{-}\right) .
$$

Here, $\hat{a}\left(\hat{a}^{\dagger}\right)$ are bosonic annihilation (creation) operators describing the main stationary mode of a cavity. The atom (with two relevant states) is coupled to this fundamental mode with strength $g$ and $\Omega$ is the amplitude of a driving field. The $\hat{\sigma}^{-}\left(\hat{\sigma}^{+}\right)$are lowering (raising) operators for the two-level system. The atom-cavity is coupled to an EM environment, that in second quantization is given by the free Hamiltonian, $\hat{H}_{\mathrm{EM}}=\int \mathrm{d} \omega \omega \hat{b}^{\dagger}(\omega) \hat{b}(\omega)$. Taking an interaction picture with respect to the EM field, the system-bath (ancilla-field) coupling can be written as

$$
\hat{H}_{\text {coupling }}(t)=\sqrt{\frac{\kappa}{2 \pi}} \int \mathrm{d} \omega \hat{a}^{\dagger} \hat{b}(\omega) \mathrm{e}^{-i \omega t}+\text { H.c. }
$$

Limiting the integration region to frequencies in resonance with the cavity, we can safely assume the RWA. Also, assuming a pointlike interaction in space, the coupling function is flat in momentum. It is customary to introduce the time dependent operators $\hat{E}^{+}(t)=i / \sqrt{2 \pi} \int \mathrm{d} \omega \mathrm{e}^{-i \omega t} \hat{b}(\omega)$ and Hermitian conjugate. They correspond to the electric field components of the EM field. In this way, we can finally write the total Hamiltonian as

$$
\hat{H}(t)=\hat{H}_{\mathrm{ancilla}}+i \sqrt{\kappa}\left(\hat{a} \otimes \hat{E}^{-}(t)-\text { H.c. }\right) .
$$

The electric field operators can, in turn, be decomposed into in-out components [49]. The in component corresponds to the field that impinges on the system while the out component consists of a reflected part plus a radiated one due to the interaction of the EM field with the system. If we take the in state of the EM field to be the vacuum, it can be shown [50] that the evolution governed by (45) can be reduced to that of the non-Hermitian Hamiltonian

$$
-i \hat{H}_{\mathrm{eff}}(t)=-i \hat{H}_{\mathrm{ancilla}}-\frac{1}{2} \kappa \hat{a}^{\dagger} \hat{a}+\sqrt{\kappa} \hat{a} \otimes \hat{E}^{-}(t) .
$$

This is the same kind of evolution that generates the cMPS ansatz [see Eqs. (1) and (6)] once we trace over the degrees of freedom of the ancilla. We thus make the following identification:

$$
R=\sqrt{\frac{\kappa}{c}} \hat{a} \quad K=\frac{1}{c} \hat{H}_{\text {ancilla }}(g, \Omega)
$$

for $x=c t$. While we do not have control over $R$, we can modify the variational parameter $K$ by properly tuning the couplings $(g, \Omega)$ of the cavity-atom system. The continuous field $\hat{\psi}(x)$ will map into the output field operators of the electromagnetic field: $\hat{\psi}(x)=\hat{E}^{+}(t) / \sqrt{c}$. Being the EM field in a cMPS state, computing expectation values of operators will translate into measuring correlations of the EM field itself, i.e., measuring the normalized correlation functions $g^{(1)}\left(t, t^{\prime}\right)$, $g^{(2)}\left(t, t^{\prime}\right)$

$$
\begin{aligned}
g^{(1)}\left(t, t^{\prime}\right) & =\frac{\left\langle\hat{E}^{-}(t) \hat{E}^{+}\left(t^{\prime}\right)\right\rangle}{\sqrt{\left\langle\hat{E}^{-}(t) \hat{E}^{+}(t)\right\rangle\left\langle\hat{E}^{-}\left(t^{\prime}\right) \hat{E}^{+}\left(t^{\prime}\right)\right\rangle}}, \\
g^{(2)}\left(t, t^{\prime}\right) & =\frac{\left\langle\hat{E}^{-}(t) \hat{E}^{-}\left(t^{\prime}\right) \hat{E}^{+}(t) \hat{E}^{+}\left(t^{\prime}\right)\right\rangle}{\left\langle\hat{E}^{-}(t) \hat{E}^{+}(t)\right\rangle\left\langle\hat{E}^{-}\left(t^{\prime}\right) \hat{E}^{+}\left(t^{\prime}\right)\right\rangle},
\end{aligned}
$$

and higher orders depending on the model we wish to simulate. Following our previous identification, the correlators $\left\langle\hat{E}^{-}(t) \hat{E}^{+}\left(t^{\prime}\right)\right\rangle$ and $\left\langle\hat{E}^{-}(t) \hat{E}^{-}\left(t^{\prime}\right) \hat{E}^{+}(t) \hat{E}^{+}\left(t^{\prime}\right)\right\rangle$ map to $\left\langle\hat{\psi}^{\dagger}(x) \hat{\psi}\left(x^{\prime}\right)\right\rangle$ and $\left\langle\hat{\psi}^{\dagger}(x) \hat{\psi}^{\dagger}\left(x^{\prime}\right) \hat{\psi}(x) \hat{\psi}\left(x^{\prime}\right)\right\rangle$, respectively. It was shown numerically that the atom-cavity setup could simulate the Lieb-Liniger model giving correlations acceptably well [32].

With this work at hand, our proposal has also a natural realization. In our case, we envision two superconducting cavities interacting each one with one [51-53] or several superconducting qubits (Fig. 5):

$$
\begin{aligned}
\hat{H}_{\text {sys }}= & \sum_{\alpha}\left(g \hat{a}_{\alpha}^{\dagger} \hat{\sigma}_{\alpha}^{-}+J \hat{a}_{\alpha}^{\dagger} \hat{a}_{\alpha+1}+\lambda \hat{\sigma}_{\alpha}^{+} \hat{\sigma}_{\alpha+1}^{-}+\right.\text {H.c. } \\
& \left.+\Omega\left(\hat{\sigma}_{\alpha}^{+}+\hat{\sigma}_{\alpha}^{-}\right)\right) .
\end{aligned}
$$

Here, $g$ is the coupling strength between a cavity and a qubit at the same site, $J$ and $\lambda$ are the coupling strengths for cavities and qubits at different sites respectively, and $\Omega$ is the amplitude

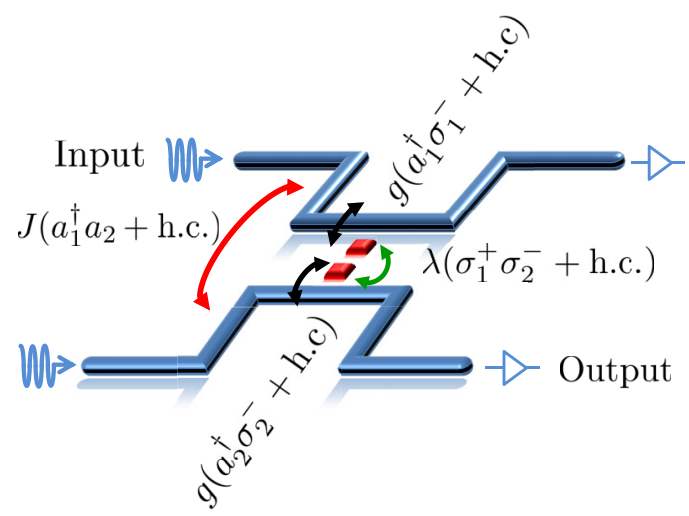

FIG. 5. (Color online) Possible circuit QED implementation for the quantum simulation of a cMPS for two coupled fields. The ancillas consist on two cavity-qubit setups and the fields are the input and output of the EM field. The Hamiltonian of the cavity-qubit setups simulates $\tilde{K}$ and the cavity operators couple to the external field, that is, they correspond to the matrices $\tilde{R}_{\alpha}$. 
of the on-site driving field. That are coupled to different baths (different fields) through

$$
\hat{H}_{\text {coupling }}(t)=\sum_{\alpha} \sqrt{\frac{\kappa_{\alpha}}{2 \pi}} \int \mathrm{d} \omega \hat{a}_{\alpha}^{\dagger} \hat{b}_{\alpha}(\omega) \mathrm{e}^{-i \omega t}+\text { H.c. }
$$

Therefore in our case $(\alpha=1,2)$, the identifications are the following:

$$
\tilde{R}_{\alpha}=\sqrt{\frac{\kappa_{\alpha}}{c}} \hat{a}_{\alpha}
$$

and

$$
\widetilde{K}=\frac{1}{c} \hat{H}_{\text {sys }}
$$

with

$$
\begin{gathered}
K_{\alpha}=\hat{H}_{\mathrm{JC}, \alpha}=\frac{g}{c}\left(\hat{a}_{\alpha}^{\dagger} \hat{\sigma}_{\alpha}^{-}+\text {H.c. }\right)+\frac{\Omega}{c}\left(\hat{\sigma}_{\alpha}^{+}+\hat{\sigma}_{\alpha}^{-}\right), \\
Z_{1}^{(1)} \otimes Z_{2}^{(1)}+Z_{1}^{(2)} \otimes Z_{2}^{(2)}=\frac{J}{c} \hat{a}_{1} \otimes \hat{a}_{2}^{\dagger}+\text { H.c. }
\end{gathered}
$$

and

$$
Z_{1}^{(3)} \otimes Z_{2}^{(3)}+Z_{1}^{(4)} \otimes Z_{2}^{(4)}=\frac{\lambda}{c} \hat{\sigma}_{1}^{+} \otimes \hat{\sigma}_{2}^{-}+\text {H.c. }
$$

Note that in Sec. IV we demanded that the matrices $Z$ should be Hermitian. Equation (55) and (56) can always be brought into a sum of tensor products of Hermitian operators. The former equations are of the form $C=A \otimes B^{\dagger}+A^{\dagger} \otimes B$. We can split any operator in terms of its Hermitian components. In the case of $A$, the decomposition reads: $A=A_{r}+i A_{i}$ (and similarly for $B)$. Here, $A_{r}=1 / 2\left(A^{\dagger}+A\right)$ and $A_{i}=i / 2\left(A^{\dagger}-\right.$
$A)$. It is straightforward to show that $C$ can be rewritten as $C=2 A_{r} \otimes B_{r}+2 A_{i} \otimes B_{i}$.

Finally, as for the single field, EM field correlations need to be computed. In addition, cross-correlations, for instance, $\left\langle\hat{E}_{i}^{-}(t) \hat{E}_{j}^{+}\left(t^{\prime}\right)\right\rangle$ will be necessary. In circuit QED, this is possible as reported in the literature [54-58].

\section{SUMMARY AND CONCLUSIONS}

In this work, we have proposed an extension of continuous matrix product states (cMPS) to study the ground-state properties of 1D coupled fields. Our treatment has been confronted to previous DMRG numerical results, showing good convergence properties even for moderately large coupling strengths. Finally, we have discussed how it could be possible to realize computations for coupled fields using a quantum simulator to implement the cMPS ansatz and optimizing over the ansatz parameters [32]. We believe that extensions of this ansatz, together with new ideas on time evolution and the study of quasiparticle excitations [23,59] can provide a valuable insight on existing experiments with $1 \mathrm{D}$ atomic Bose-Einstein condensates $[21,60]$.

\section{ACKNOWLEDGMENTS}

Discussions with Diego Blas and Sergey Sibiryakov are appreciated. We acknowledge support from the Spanish DGICYT under Project Nos. FIS2011-25167 and FIS201233022, by the Aragon (Grupo FENOL) and the EU Project PROMISCE. The authors would also like to acknowledge the Centro de Ciencias de Benasque Pedro Pascual for its hospitality.
[1] K. G. Wilson, Rev. Mod. Phys. 47, 773 (1975).

[2] S. R. White, Phys. Rev. Lett. 69, 2863 (1992).

[3] U. Schollwöck, Rev. Mod. Phys. 77, 259 (2005).

[4] F. Verstraete, D. Porras, and J. I. Cirac, Phys. Rev. Lett. 93, 227205 (2004).

[5] G. Vidal, Phys. Rev. Lett. 93, 040502 (2004).

[6] F. Verstraete, J. J. García-Ripoll, and J. I. Cirac, Phys. Rev. Lett. 93, 207204 (2004).

[7] S. R. White and A. E. Feiguin, Phys. Rev. Lett. 93, 076401 (2004).

[8] A. J. Daley, C. Kollath, U. Schollwöck, and G. Vidal, J. Stat. Mech. (2004) P04005.

[9] J. J. García-Ripoll, New J. Phys. 8, 305 (2006).

[10] G. Vidal, Phys. Rev. Lett. 99, 220405 (2007).

[11] M. C. Bañuls, D. Pérez-García, M. M. Wolf, F. Verstraete, and J. I. Cirac, Phys. Rev. A 77, 052306 (2008).

[12] M. Cheneau, P. Barmettler, D. Poletti, M. Endres, P. Schauß, T. Fukuhara, C. Gross, I. Bloch, C. Kollath, and S. Kuhr, Nature (London) 481, 484 (2012).

[13] S. Trotzky, Y.-A. Chen, A. Flesch, I. P. McCulloch, U. Schollwöck, J. Eisert, and I. Bloch, Nat. Phys. 8, 325 (2012).

[14] T. Fukuhara, A. Kantian, M. Endres, M. Cheneau, P. Schauß, S. Hild, D. Bellem, U. Schollwöck, T. Giamarchi, C. Gross et al., Nat. Phys. 9, 235 (2013).

[15] P. Hauke and L. Tagliacozzo, Phys. Rev. Lett. 111, 207202 (2013).
[16] B. Peropadre, D. Zueco, D. Porras, and J. J. García-Ripoll, Phys. Rev. Lett. 111, 243602 (2013).

[17] E. Sánchez-Burillo, D. Zueco, J. García-Ripoll, and L. MartínMoreno, arXiv:1406.5779 [quant-ph].

[18] T. Giamarchi, Quantum Physics in One Dimension, International Series of Monographs on Physics (Clarendon Press, Oxford, 2004).

[19] M. A. Cazalilla, R. Citro, T. Giamarchi, E. Orignac, and M. Rigol, Rev. Mod. Phys. 83, 1405 (2011).

[20] A. Steffens, M. Friesdorf, T. Langen, B. Rauer, T. Schweigler, R. Hübener, J. Schmiedmayer, C. A. Riofrío, and J. Eisert, arXiv:1406.3632.

[21] A. Steffens, C. A. Riofrío, R. Hübener, and J. Eisert, arXiv:1406.3631.

[22] F. Verstraete and J. I. Cirac, Phys. Rev. Lett. 104, 190405 (2010).

[23] J. Haegeman, J. I. Cirac, T. J. Osborne, and F. Verstraete, Phys. Rev. B 88, 085118 (2013)

[24] D. Draxler, J. Haegeman, T. J. Osborne, V. Stojevic, L. Vanderstraeten, and F. Verstraete, Phys. Rev. Lett. 111, 020402 (2013)

[25] J. Haegeman, J. I. Cirac, T. J. Osborne, H. Verschelde, and F. Verstraete, Phys. Rev. Lett. 105, 251601 (2010)

[26] V. Stojevic, J. Haegeman, I. P. McCulloch, L. Tagliacozzo, and F. Verstraete, arXiv:1401.7654.

[27] M. A. Cazalilla, A. F. Ho, and T. Giamarchi, New J. Phys. 8, 158 (2006). 
[28] F. D. M. Haldane, J. Phys. C. 14, 2585 (1981).

[29] F. D. M. Haldane, Phys. Rev. Lett. 47, 1840 (1981).

[30] M. A. Cazalilla, J. Phys. B 37, S1 (2004).

[31] E. Orignac, M. Tsuchiizu, and Y. Suzumura, Phys. Rev. A 81, 053626 (2010).

[32] S. Barrett, K. Hammerer, S. Harrison, T. E. Northup, and T. J. Osborne, Phys. Rev. Lett. 110, 090501 (2013).

[33] C. Brockt, J. Haegeman, D. Jennings, T. J. Osborne, and F. Verstraete, arXiv:1210.5401.

[34] M. Srednicki, Phys. Rev. Lett. 71, 666 (1993).

[35] T. J. Osborne, J. Eisert, and F. Verstraete, Phys. Rev. Lett. 105, 260401 (2010).

[36] M. Rispler, Master's thesis, Imperial College of London, 2012.

[37] A. Rivas and S. F. Huelga, Open Quantum Systems. An Introduction, Springer Briefs in Physics (Springer, Berlin, Heidelberg, 2011).

[38] E. H. Lieb and W. Liniger, Phys. Rev. 130, 1605 (1963).

[39] E. H. Lieb, Phys. Rev. 130, 1616 (1963).

[40] M. A. Cazalilla and A. F. Ho, Phys. Rev. Lett. 91, 150403 (2003).

[41] L. Mathey, Phys. Rev. B 75, 144510 (2007).

[42] A. Kleine, C. Kollath, I. P. McCulloch, T. Giamarchi, and U. Schollwöck, New J. Phys. 10, 045025 (2008).

[43] A. Kleine, Ph.D. thesis, RWTH Aachen University, 2010.

[44] I. M. Georgescu, S. Ashhab, and F. Nori, Rev. Mod. Phys. 86, 153 (2014).

[45] C. Schön, E. Solano, F. Verstraete, J. I. Cirac, and M. M. Wolf, Phys. Rev. Lett. 95, 110503 (2005).

[46] C. Schön, K. Hammerer, M. M. Wolf, J. I. Cirac, and E. Solano, Phys. Rev. A 75, 032311 (2007).

[47] A. Wallraff, D. I. Schuster, A. Blais, L. Frunzio, R.-S. Huang, J. Majer, S. Kumar, S. M. Girvin, and R. J. Schoelkopf, Nature (London) 431, 162 (2004).

[48] J. Q. You and F. Nori, Nature (London) 474, 589 (2011).
[49] C. W. Gardiner and M. J. Collett, Phys. Rev. A 31, 3761 (1985).

[50] C. Gardiner and P. Zoller, Quantum Noise: A Handbook of Markovian and Non-Markovian Quantum Stochastic Methods with Applications to Quantum Optics, Springer Series in Synergetics (Springer, Berlin, Heidelberg, 2004).

[51] L. Sun, A. Petrenko, Z. Leghtas, B. Vlastakis, G. Kirchmair, K. M. Sliwa, A. Narla, M. Hatridge, S. Shankar, J. Blumoff, L. Frunzio, M. Mirrahimi, M. H. Devoret, and R. J. Schoelkopf, Nature (London) 511, 444 (2014).

[52] M. Mariantoni, F. Deppe, A. Marx, R. Gross, F. K. Wilhelm, and E. Solano, Phys. Rev. B 78, 104508 (2008).

[53] G. M. Reuther, D. Zueco, F. Deppe, E. Hoffmann, E. P. Menzel, T. Weiß1, M. Mariantoni, S. Kohler, A. Marx, E. Solano, R. Gross, and P. Hänggi, Phys. Rev. B 81, 144510 (2010).

[54] C. Eichler, D. Bozyigit, C. Lang, L. Steffen, J. Fink, and A. Wallraff, Phys. Rev. Lett. 106, 220503 (2011).

[55] R. Di Candia, E. P. Menzel, L. Zhong, F. Deppe, A. Marx, R. Gross, and E. Solano, New J. Phys. 16, 015001 (2014).

[56] E. P. Menzel, F. Deppe, M. Mariantoni, M. A. Araque Caballero, A. Baust, T. Niemczyk, E. Hoffmann, A. Marx, E. Solano, and R. Gross, Phys. Rev. Lett. 105, 100401 (2010).

[57] D. Bozyigit, C. Lang, L. Steffen, J. M. Fink, C. Eichler, M. Baur, R. Bianchetti, P. J. Leek, S. Filipp, M. P. da Silva, A. Blais, and A. Wallraff, Nat. Phys. 7, 154 (2010).

[58] D. Bozyigit, C. Lang, L. Steffen, J. M. Fink, C. Eichler, M. Baur, R. Bianchetti, P. J. Leek, S. Filipp, A. Wallraff, M. P. Da Silva, and A. Blais, J. Phys.: Conf. Ser. 264, 012024 (2011).

[59] J. Haegeman, S. Michalakis, B. Nachtergaele, T. J. Osborne, N. Schuch, and F. Verstraete, Phys. Rev. Lett. 111, 080401 (2013).

[60] T. Langen, R. Geiger, M. Kuhnert, B. Rauer, and J. Schmiedmayer, Nat. Phys. 9, 640 (2013). 